\title{
Exponential Lomax Distribution
}

\author{
A. H. El-Bassiouny \\ Mansoura University \\ Department of Mathematics, \\ Faculty of Science, 35516 \\ Mansoura, Egypt
}

\author{
N. F. Abdo \\ Mansoura University \\ Department of Mathematics, \\ Faculty of Science, 35516 \\ Mansoura, Egypt
}

\author{
H. S. Shahen \\ Mansoura University \\ Department of Mathematics, \\ Faculty of Science, 35516 \\ Mansoura, Egypt
}

\begin{abstract}
In this paper, A new distribution called Exponential Lomax distribution is introduced. It is seemed that the parameter values of our new distribution are depending on decreasing and upside-down bathtub failure rate function. Also, the statistical properties of this model are studied, such as, quantiles, moments, mean deviation. Moreover, maximum likelihood estimators of it's parameters are discussed. Finally, the procedure is illustrated by real data set. It is shown that the introduced model is more competitive than other models.
\end{abstract}

\section{Keywords}

Exponential Lomax distribution, Lomax distribution, moments, quantiles and Maximum likelihood estimation.

\section{INTRODUCTION}

The Lomax or Pareto II (the shifted Pareto) distribution was proposed by Lomax [24]. This distribution has found wide applications such as the analysis of the business failure life time data, income and wealth inequality, size of cities, actuarial science, medical and biological sciences, engineering, lifetime and reliability modeling. Furthermore, it has been applied to model data obtained from income and wealth by Harris [17] and Atkinson et al. [5], firm size by Corbellini et al. [10], size distribution of computer files on servers by Hollanh et al. [19], reliability and life testing by Hassan et al. [18], receiver operating characteristic (ROC) curve analysis by Campbell et al. [9] and Hirsch-related statistics by Glanzel [15]. In the lifetime, the Lomax model belongs to the family of decreasing failure rate by Chahkandi et al. [11] and arises as a limiting distribution of residual lifetimes at great age by Balkema et al. [7]. This distribution has been proposed as heavy tailed alternative to the exponential, Weibull and gamma distributions by Bryson [8]. Moreover, it is related to the Burr family of distributions as in Tadikamalla [27]. Durbey [13] proved that it can be obtained as a special case from compound gamma distributions. Some details about the Lomax distribution and Pareto family are given by Arnold [4] and Johnson et al. [20]. Some properties and moments for the Lomax distribution have been discussed by Ahsanullah [2], Amin [3], Balakrishnan [6] and Lee et al. [22]. In the literature, some extensions of the Lomax distribution are available such as the exponentiated Lomax (EL) by Abdul-Moniem [1], Marshall-Olkin extended-Lomax (MOEL) by Ghitany et al. [14] and Gupta et al [16], betaLomax (BL), Kumaraswamy-Lomax (KwL), McDonaldLomax (McL) by Lemonte et al. [23] and gamma-Lomax (GL) by Cordeiro et al. [12].

In this article, A new generalization of the lomax distribution called Exponential lomax (ELomax) distribution is introduced in Section 2. Various properties including quantiles and the hazard function are investigated in Section 3. The moments of the ELomax are introduced in Section 4. The mean deviation from the mean and the mean deviation from the median are provided in Section 5. Section 6 is devoted to the maximum likelihood estimates of the parameters of the ELomax. In Section 7, an application of the ELomax to an actual Floyd river flood data set is provided.

\section{THE EXPONENTIAL LOMAX DISTRIBUTION}

If we have $c d f G(x)$ of any random variable $X$ then the function

$F(x)=\int_{0}^{\frac{1}{1-G(x)}} \lambda e^{-\lambda x} d x, \lambda>0$

is called an exponential distribution see [17].

The Lomax distribution is introduced by the following form:

$G(x ; \alpha, \beta)=1-\left(\frac{\beta}{x+\beta}\right)^{\alpha}, x>0, \alpha, \beta>0$,

where $\beta$ is a scale parameter, and $\alpha$ is a shape parameter. Using the form in (1), then the $c d f$ for the Exponential Lomax (ELomax) distribution, for $\gamma>0$, is given by

$$
\begin{aligned}
& \frac{1}{\left(\frac{\beta}{x+\beta}\right)^{\alpha}} \\
& F(x)=\int_{0} \lambda e^{-\lambda x} d x, \alpha, \beta, \lambda>0, \\
& =1-e^{-\lambda\left(\frac{\beta}{x+\beta}\right)^{-\alpha}} \text {. }
\end{aligned}
$$

Hence, the corresponding $p d f$ for $F(x)$, is given by

$f(x)=\frac{\alpha \lambda}{\beta}\left(\frac{\beta}{x+\beta}\right)^{-\alpha+1} e^{-\lambda\left(\frac{\beta}{x+\beta}\right)^{-\alpha}}, x \geq-\beta, \alpha, \beta, \lambda>0$.

Figures 1 and 2 illustrate some of the possible shapes of the $p d f$ of the ELomax distribution.

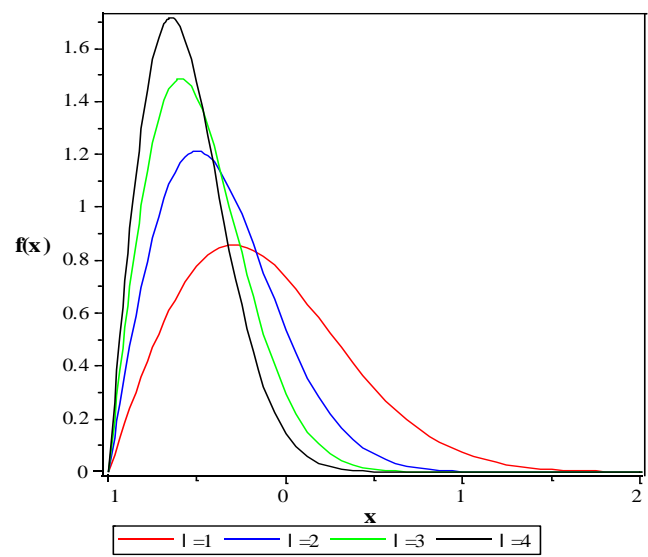

Fig 1. Plot of the $p d f$ for different values of $\lambda$ for $\alpha=2$ and $\beta=1$. 


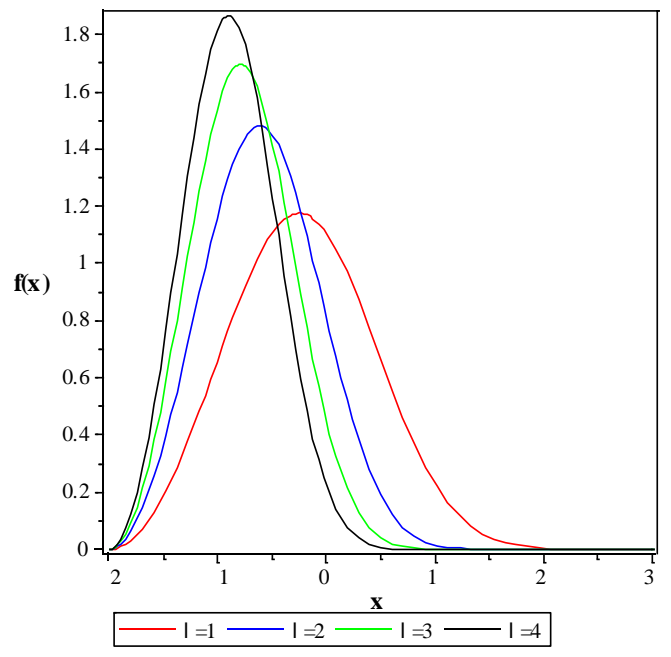

Fig 2. Plot of the $p d f$ for different values of $\lambda$ for $\alpha=3$ and $\beta=2$.

Figures 3 and 4 illustrates some of the possible shapes of the $c d f$ of the ELomax distribution.

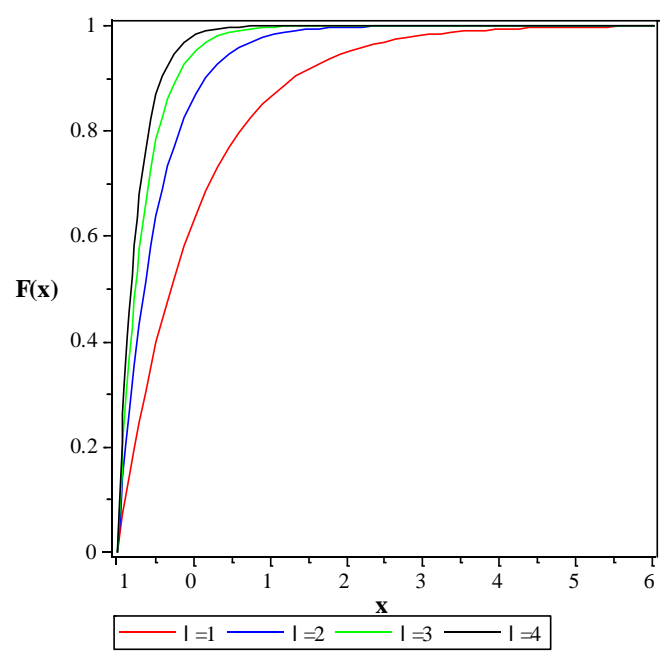

Fig 3. Plot of the $c d f$ for different values of $\lambda$ for $\alpha=1$ and $\beta=1$.

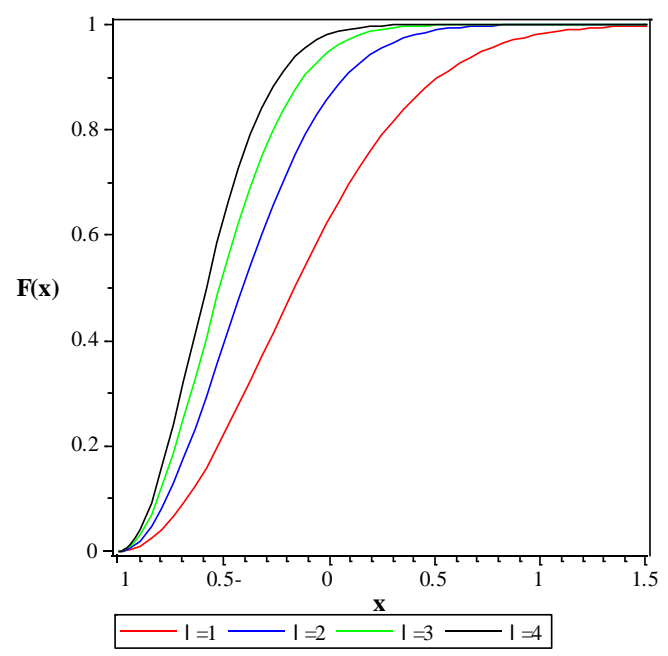

Fig 4. Plot of the $c d f$ for different values of $\lambda$ for $\alpha=2$ and $\beta=1$.

\section{PROPERTIES OF THE ELOMAX}

In this section, we discuss some properties of the ELomax distribution.

\subsection{Quantiles}

The qth quantile $\left(x_{q}\right)$ and the median $\left(x_{0.5}\right)$ of the ELomax distribution respectively, are given by

$$
\begin{aligned}
& x_{q}=F^{-1}(q)=\beta\left[\left(\frac{-\ln (1-q)}{\lambda}\right)^{\frac{1}{\alpha}}-1\right] . \\
& \operatorname{median}(X)=x_{0.5}=\beta\left[\left(\frac{-\ln (0.5)}{\lambda}\right)^{\frac{1}{\alpha}}-1\right] .
\end{aligned}
$$

\subsection{Hazard rate function}

The hazard rate function $h(x)$ and the reversed hazard rate function $\tau(x)$ are an important quantities characterizing life phenomena. For the ELomax distribution, $h(x)$ and $\tau(x)$ are given by the following theorem:

\section{Theorem 1}

The hazard rate function and reversed hazard rate function for the ELomax distribution are given

$$
\begin{aligned}
h(x) & =\frac{\frac{\lambda \alpha}{\beta}\left(\frac{\beta}{x+\beta}\right)^{-\alpha+1} e^{-\lambda\left(\frac{\beta}{x+\beta}\right)^{-\alpha}}}{e^{-\lambda\left(\frac{\beta}{x+\beta}\right)^{-\alpha}}} \\
& =\frac{\lambda \alpha}{\beta}\left(\frac{\beta}{x+\beta}\right)^{-\alpha+1} \\
\tau(x) & =\frac{\frac{\lambda \alpha}{\beta}\left(\frac{\beta}{x+\beta}\right)^{-\alpha+1} e^{-\lambda\left(\frac{\beta}{x+\beta}\right)^{-\alpha}}}{1-e^{-\lambda\left(\frac{\beta}{x+\beta}\right)^{-\alpha}}}
\end{aligned}
$$

Proof

This can be easily deduced by using

$$
h(x)=\frac{f(x)}{1-F(x)}, \tau(x)=\frac{f(x)}{F(x)}
$$

Figures 5 and 6 illustrate some of the possible shapes of the hazard rate function of the ELomax distribution.

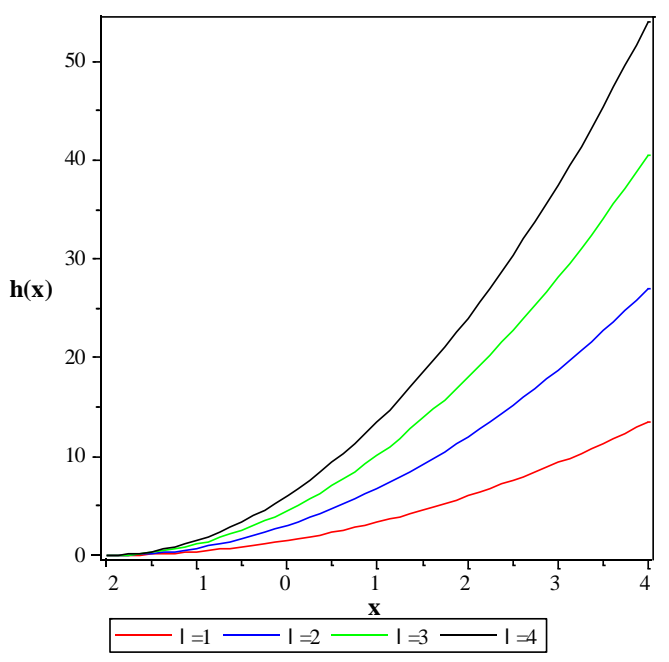

Fig 5. Plot of the hazard rate function for different values of $\lambda$ for $\alpha=3$ and $\beta=2$. 


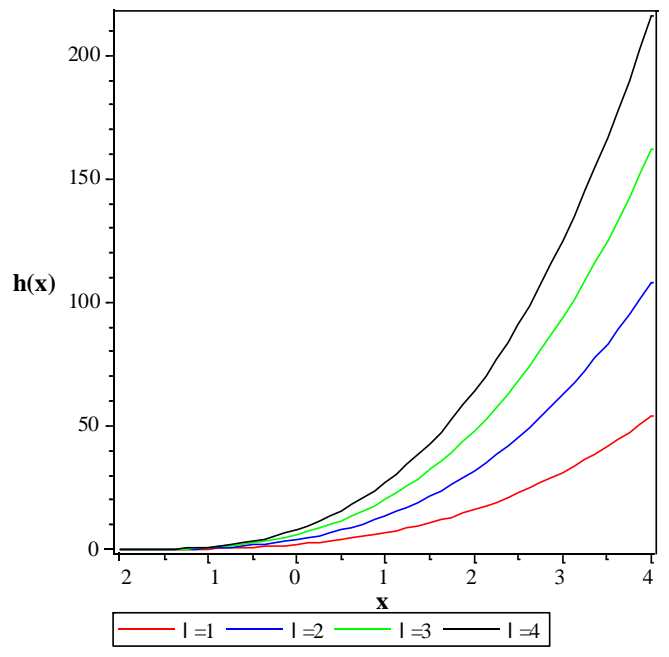

Fig 5. Plot of the hazard rate function for different values of $\lambda$ for $\alpha=4$ and $\beta=2$.

\section{MOMENTS OF THE ELOMAX}

In this section, the rth moment about the mean and the rth moment about the origin of the ELomax distribution are computed.

\section{Theorem 2}

The rth moment about the mean of the ELomax distribution is as follows :

$E(X-\mu)^{r}=\sum_{j=0}^{r}\left(\begin{array}{l}r \\ j\end{array}\right)\left(\frac{\beta}{\sqrt[\alpha]{\lambda}}\right)^{j}(-\mu-\beta)^{r-j} \Gamma\left(\frac{j}{\alpha}+1\right)$,

$r=1,2,3, \ldots$ and the rth moment about the origin is

$$
E(X)^{r}=\sum_{j=0}^{r}\left(\begin{array}{l}
r \\
j
\end{array}\right)\left(\frac{\beta}{\sqrt[\alpha]{\lambda}}\right)^{j}(-\beta)^{r-j} \Gamma\left(\frac{j}{\alpha}+1\right), r=1,2,3, \ldots
$$

\section{Proof}

The rth moment about the mean is given by

$$
\begin{aligned}
& E(X-\mu)^{r}=\int_{-\infty}^{\infty}(x-\mu)^{r} f(x) d x \\
& =\int_{-\beta}^{\infty}(x-\mu)^{r} \frac{\lambda \alpha}{\beta}\left(\frac{\beta}{x+\beta}\right)^{-\alpha+1} e^{-\lambda\left(\frac{\beta}{x+\beta}\right)^{-\alpha}} d x,
\end{aligned}
$$

let $u=\lambda\left(\frac{\beta}{x+\beta}\right)^{-\alpha}$, then $d x=\frac{\beta}{\alpha(\sqrt[\alpha]{\lambda})} u^{\left(\frac{1}{\alpha}-1\right)} d u, u(-\beta)=0$ and $u(\infty)=\infty$, also

$$
\begin{aligned}
x=\frac{\beta}{(\sqrt[\alpha]{\lambda})} u^{\left(\frac{1}{\alpha}\right)}-\beta, \text { then } & \\
E(X-\mu)^{r} & =\int_{0}^{\infty}\left(\frac{\beta}{\sqrt[\alpha]{\lambda}} u^{\left(\frac{1}{\alpha}\right)}-\beta-\mu\right)^{r} e^{-u} d u \\
= & \sum_{j=0}^{r}\left(\begin{array}{l}
r \\
j
\end{array}\right)\left(\frac{\beta}{\sqrt[\alpha]{\lambda}}\right)^{j}(-\mu-\beta)^{r-j} \int_{0}^{\infty} u^{\left(\frac{j}{\alpha}\right)} e^{-u} d u \\
& =\sum_{j=0}^{r}\left(\begin{array}{l}
r \\
j
\end{array}\right)\left(\frac{\beta}{\sqrt[\alpha]{\lambda}}\right)^{j}(-\mu-\beta)^{r-j} \Gamma\left(\frac{j}{\alpha}+1\right) .
\end{aligned}
$$

Thus the rth moment about the mean is

$$
\begin{gathered}
E(X-\mu)^{r}=\sum_{j=0}^{r}\left(\begin{array}{l}
r \\
j
\end{array}\right)\left(\frac{\beta}{\sqrt[\alpha]{\lambda}}\right)^{j}(-\mu-\beta)^{r-j} \Gamma\left(\frac{j}{\alpha}+1\right), r=1, \\
2,3, \ldots,
\end{gathered}
$$

where $E(X)=\mu$ then If $\mu=0$. Hence the rth moment about the origin is

$$
E(X)^{r}=\sum_{j=0}^{r}\left(\begin{array}{l}
r \\
j
\end{array}\right)\left(\frac{\beta}{\sqrt[\alpha]{\lambda}}\right)^{j}(-\beta)^{r-j} \Gamma\left(\frac{j}{\alpha}+1\right), r=1,2,3, \ldots
$$

Using the appropriate moment expressions, the variance is given as follows:

$$
\begin{gathered}
\operatorname{var}(X)=E\left(X^{2}\right)-E^{2}(X) \\
=\sum_{j=0}^{2}\left(\begin{array}{l}
2 \\
j
\end{array}\right)\left(\frac{\beta}{\sqrt[\alpha]{\lambda}}\right)^{j}(-\beta)^{2-j} \Gamma\left(\frac{j}{\alpha}+1\right)- \\
{\left[\sum_{j=0}^{1}\left(\begin{array}{l}
1 \\
j
\end{array}\right)\left(\frac{\beta}{\sqrt[\alpha]{\lambda}}\right)^{j}(-\beta)^{-j} \Gamma\left(\frac{j}{\alpha}+1\right),\right]^{2}}
\end{gathered}
$$

The skewness and kurtosis can now be calculated by using the relations

Skewness $=\frac{E\left(X^{3}\right)-3 E(X) E\left(X^{2}\right)+2 E^{3}(X)}{\operatorname{var}^{3 / 2}(X)}$

Kurtosis $=\frac{E\left(X^{4}\right)-4 E(X) E\left(X^{3}\right)+6 E\left(X^{2}\right) E^{2}(X)-3 E^{4}(X)}{\operatorname{var}^{2}(X)}$

\section{THE MEAN DEVIATION OF THE ELOMAX}

The amount of spread in a population is evidently measured to some extent by the totality of deviations from the mean and median. These are known as the mean deviation about the mean and the mean deviation about the median. Let $X$ be an Exponential Lomax random variable with mean $E(X)=\mu$ and median $m$.

\section{Theorem 3}

The mean deviation from the mean of the ELomax distribution is as follows :

$$
\begin{gathered}
D(\mu)=2(\mu-d)-2 \sum_{i=0}^{\infty}\left(\frac{-\lambda}{\beta^{\alpha}}\right)^{i} \frac{1}{i !(\alpha i+1)}\left((\mu+\beta)^{\alpha i+1}-\right. \\
\left.(d+\beta)^{\alpha i+1}\right)
\end{gathered}
$$

Proof

$$
\begin{aligned}
D(\mu) & =E(|X-\mu|)=\int_{d}^{\infty}|X-\mu| f(x) d x \\
& =\int_{d}^{\mu}(\mu-x) f(x) d x+\int_{\mu}^{\infty}(x-\mu) f(x) d x \\
& =2 \int_{d}^{\mu}(\mu-x) f(x) d x \\
& =2 \mu f(\mu)-2 \int_{d}^{\mu} x f(x) d x \\
& =2 \mu f(\mu)-2 \int_{d}^{\mu} x d f(x)=2 \int_{d}^{\mu} F(x) d x \\
& =2(\mu-d)-2 \int_{d}^{\mu} e^{-\lambda\left(\frac{\beta}{x+\beta}\right)^{-\alpha}} d x
\end{aligned}
$$




$$
\begin{gathered}
=2(\mu-d)-2 \sum_{i=0}^{\infty}\left(\frac{-\lambda}{\beta^{\alpha}}\right)^{i} \frac{1}{i !} \int_{d}^{\mu}(x+\beta)^{\alpha i} d x \\
=2(\mu-d)-2 \sum_{i=0}^{\infty}\left(\frac{-\lambda}{\beta^{\alpha}}\right)^{i} \frac{1}{i !(\alpha i+1)}\left((\mu+\beta)^{\alpha i+1}\right. \\
\left.-(d+\beta)^{\alpha i+1}\right)
\end{gathered}
$$

\section{Theorem 3}

The mean deviation from the median of the ELomax distribution is as follows :

$$
\begin{aligned}
D(m)= & \mu+m-2 d-2 \sum_{i=0}^{\infty}\left(\frac{-\lambda}{\beta^{\alpha}}\right)^{i} \frac{1}{i !(\alpha i+1)}\left((m+\beta)^{\alpha i+1}-\right. \\
& \left.(d+\beta)^{\alpha i+1}\right)
\end{aligned}
$$

Proof

$$
\begin{aligned}
D(m) & =E(|X-m|)=\int_{d}^{\infty}|X-m| f(x) d x \\
& =2 \int_{d}^{m}(m-x) f(x) d x+\int_{d}^{\infty}(x-m) f(x) d x \\
& =2 m F(m)-2 \int_{d}^{m} x f(x) d x+E(X-m) \\
& =2 m F(m)-2 \int_{d}^{m} x f(x) d x+\mu-m \\
& =\mu-m+2 \int_{d}^{m} F(x) d x \\
=\mu+m-2 d-2 \sum_{i=0}^{\infty}\left(\frac{-\lambda}{\beta^{\alpha}}\right)^{i} \frac{1}{i !(\alpha i+1)}\left((m+\beta)^{\alpha i+1}\right. & \left.-(d+\beta)^{\alpha i+1}\right)
\end{aligned}
$$

\section{MAXIMUM LIKELIHOOD ESTIMATES OF THE PARAMETERS}

Let $x_{1}, x_{2}, \ldots, x_{n}$ be a random sample from Exponential Lomax distribution. Then the log-likelihood function of the Exponential Lomax may be expressed as

$$
\begin{aligned}
& \log L(x ; \alpha, \beta, \lambda)=n \log \alpha+n \log \lambda-n \log \beta+ \\
& \quad(-\alpha+1) \sum_{i=1}^{n} \log \left(\frac{\beta}{x_{i}+\beta}\right)-\lambda \sum_{i=1}^{n}\left(\frac{\beta}{x_{i}+\beta}\right)^{-\alpha}
\end{aligned}
$$

Differentiating the log-likelihood with respect to, $\lambda$ and $\alpha$, respectively, and setting the result equal to zero, we have

$$
\begin{gathered}
\frac{\partial \log L(x)}{\partial \lambda}=\frac{n}{\lambda}-\sum_{i=1}^{n}\left(\frac{\beta}{x_{i}+\beta}\right)^{-\alpha} \\
\frac{\partial \log L(x)}{\partial \alpha}=\frac{n}{\alpha}-\sum_{i=1}^{n} \log \left(\frac{\beta}{x_{i}+\beta}\right)+\lambda \sum_{i=1}^{n}\left(\frac{\beta}{x_{i}+\beta}\right)^{-\alpha} \log \left(\frac{\beta}{x_{i}+\beta}\right)
\end{gathered}
$$

The maximum likelihood estimates $\hat{\lambda}$ and $\hat{\alpha}$ of the unknown parameters $\lambda$ and $\alpha$ respectively, are obtained by solving Equations (17) - (18). Since the support of the random variable $X$ depends on the parameter $\beta$, then we can't differentiate the $\log L(x ; \alpha, \beta, \lambda)$ with respect to $\beta$. In order to get it's MLE we define the two statistics $y_{1}=$ $\min \left(x_{1}, x_{2}, \ldots, x_{n}\right)$ and $y_{n}=\max \left(x_{1}, x_{2}, \ldots, x_{n}\right)$ hence the MLE of $\beta$ is any statistic in the interval $\left[-y_{1}, \infty\right)$. In order to maximize the $\log L(x ; \alpha, \beta, \lambda)$ we estimate the parameter $\beta$ by the minimum value in the above interval $\left(-y_{1}\right)$ since $\beta$ is in the denominator of the log-likelihood.

\section{APPLICATIONS ON THE ELOMAX MODEL}

In this section, a real data set is used to compare the fits of the Exponential Lomax distribution with other generalization of Lomax distribution. The data set is the failure times of 84 Aircraft Windshield.

The windshield on a large aircraft is a complex piece of equipment, comprised basically of several layers of material, including a very strong outer skin with a heated layer just beneath it, all laminated under high temperature and pressure. Failures of these items are not structural failures. Instead, they typically involve damage or delamination of the nonstructural outer ply or failure of the heating system. These failures do not result in damage to the aircraft but do result in replacement of the windshield. We consider the data on failure times for a particular model windshield given in Table 16.11 of Murthy et al. [20]. These data were recently studied by Ramos et al. [21].

The failure times of 84 Aircraft Windshield is

0.040, 1.866, 2.385, 3.443, 0.301, 1.876, 2.481, 3.467, 0.309, $1.899,2.610,3.478,0.557,1.911,2.625,3.578,0.943,1.912$ $2.632,3.595,1.070,1.914,2.646,3.699,1.124,1.981,2.661$, $3.779,1.248,2.010,2.688,3.924,1.281,2.038,2.82,3,4.035$, $1.281,2.085,2.890,4.121,1.303,2.089,2.902,4.167,1.432$, $2.097,2.934,4.240,1.480,2.135,2.962,4.255,1.505,2.154$, $2.964,4.278,1.506,2.190,3.000,4.305,1.568,2.194,3.103$, $4.376,1.615,2.223,3.114,4.449,1.619,2.224,3.117,4.485$, $1.652,2.229,3.166,4.570,1.652,2.300,3.344,4.602,1.757$, $2.324,3.376,4.663$.

The required numerical evaluations are carried out using the Package of Mathcad software. Table 1 provide the MLEs of the model parameters.The model selection is carried out using the $A I C$ (Akaike information criterion), the BIC (Bayesian information criterion) and the CAIC (consistent Akaike information criteria):

$$
\begin{gathered}
A I C=-2 l+2 q, \\
B I C=-2 l+q \log n, \\
C A I C=-2 l+\frac{2 q n}{n-q-1} .
\end{gathered}
$$

Where $l$ denotes the log-likelihood function evaluated at the maximum likelihood estimates, $q$ is the number of parameters and $n$ is the sample size. The values of the $A I C, C A I C$ and $B I C$ are listed in Table 2

Table 1. MLEs (in parentheses) for failure times of 84 Aircraft Windshield data

\begin{tabular}{|l|l|l|l|l|l|}
\hline $\begin{array}{l}\text { Distribut } \\
\text { ion }\end{array}$ & $\mathbf{a}$ & $\mathbf{b}$ & $\boldsymbol{\alpha}$ & $\boldsymbol{\beta}$ & $\boldsymbol{\lambda}$ \\
\hline ELomax & - & - & 2.448 & 0.04 & $\begin{array}{l}2.763 \mathrm{x} \\
10^{-5}\end{array}$ \\
\hline McL & $\begin{array}{l}2.18 \\
75\end{array}$ & $\begin{array}{l}119.17 \\
51\end{array}$ & 19.9243 & 75.6606 & - \\
\hline
\end{tabular}




\begin{tabular}{|l|l|l|l|l|l|}
\hline KwL & $\begin{array}{l}2.61 \\
5\end{array}$ & $\begin{array}{l}100.27 \\
56\end{array}$ & 5.2771 & 78.6774 & - \\
\hline GL & $\begin{array}{l}3.58 \\
76\end{array}$ & - & $\begin{array}{l}52001.4 \\
994\end{array}$ & $\begin{array}{l}37029.6 \\
583\end{array}$ & - \\
\hline BL & 3.60 & 33.638 & 4.8307 & $\begin{array}{l}118.837 \\
4\end{array}$ & - \\
\hline EL & 36 & 7 & $\begin{array}{l}20074.5 \\
097\end{array}$ & $\begin{array}{l}26257.6 \\
808\end{array}$ & - \\
& 61 & - & 097 & 131789. & - \\
Lomax & - & - & 51425.3 & 5 & \\
& & & 5 & &
\end{tabular}

Table 2. The statistics $l, A I C, C A I C$ and $B I C$ for failure times of 84 Aircraft Windshield data

\begin{tabular}{|l|c|c|c|c|}
\hline Distribution & \multicolumn{1}{|c|}{$\boldsymbol{A}$ IC } & $\boldsymbol{B I C}$ & $\boldsymbol{C A I C}$ \\
\hline ELomax & -129.193 & 264.386 & 264.158 & 264.686 \\
\hline McL & -129.8023 & 269.6045 & 281.8178 & 270.3640 \\
\hline KwL & -132.4048 & 272.8096 & 282.5802 & 273.3096 \\
\hline GL & -138.4042 & 282.8083 & 290.1363 & 283.1046 \\
\hline BL & -138.7177 & 285.4354 & 295.2060 & 285.9354 \\
\hline EL & -141.3997 & 288.7994 & 296.1273 & 289.0957 \\
\hline Lomax & -164.9884 & 333.9767 & 338.8620 & 334.1230 \\
\hline
\end{tabular}

Since the values of the $A I C, B I C$ and $C A I C$ are smaller for the ELomax distribution compared with those values of the other models, then the introduced distribution seems to be a very competitive model to these data.

\section{CONCLUSIONS}

In this paper, we proposed a new generalization of Lomax distribution we called it Exponential Lomax distribution. Some statistical properties of this distribution have been derived and discussed such as quantile and median. The maximum likelihood estimators of the parameters are derived. A real data set is analyzed using the new distribution, the exponentiated Lomax, Marshall-Olkin extended-Lomax, betaLomax, Kumaraswamy-Lomax, McDonald-Lomax and gamma-Lomax. Baised on the comparisons between all these models, we conclude that, the introduced model is highly competitive in the sense of fitting this real data set..

\section{REFERENCES}

[1] Abdul-Moniem, I. B. 2012. Recurrence realtions for moments of lower generalized order statistics from exponentiated Lomax distribution and its characterization, International Journal of Mathematical Archive 3, 2144国2150.
[2] Ahsanullah, M. 1991. Recoed values of the Lomax distribution, Statistica Neerlandica 45, 21?29.

[3] Amin, E. A. 2011. Kth upper record values and their moments, International Mathematical Forum 6, 3013료연.

[4] Arnold, B. C. 1983. Pareto Distributions (International Cooperative Publishing House, Maryland).

[5] Atkinson, A. B. and Harrison, A. J. 1978. Distribution of Personal Wealth in Britain (Cambridge University Press, Cambridge).

[6] Balakrishnan, N. and Ahsanullah, M. 1994. Relations for single and product moments of record values from Lomax distribution, Sankhya Series B 17, 140146

[7] Balkema, A. A. and de Hann, L. 1974. Residual life at

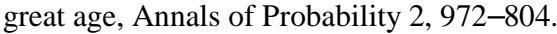

[8] Bryson, M. C. 1974. Heavy-tailed distribution: properties and tests, Technometrics 16, 161]68.

[9] Campbell, G. and Ratnaparkhi, M. V. 1993. An application of Lomax distributions in receiver operating characteristic (ROC) curve analysis, Communications in Statistics?Theory Methods 22, 1681?1697.

[10] Corbellini, A., Crosato, L., Ganugi, P and Mazzoli, M. 2007. Fitting Pareto II distributions on ??rm size: Statistical methodology and economic puzzles. Paper presented at the International Conference on Applied Stochastic Models and Data Analysis, Chania, Crete.

[11] Chahkandi, M. and Ganjali, M. 2009. On some lifetime distributions with decrasing failure rate, Computational Statistics and Data Analysis 53, 4433?2440.

[12] Cordeiro, G. M., Ortega, E. M. M. and Popovi'c, B. V. 2013. The gamma-Lomax distribution, Journal of Statistical computation and Simulation iFirst, doi:10.1080/00949655.2013.822869, 2013

[13] Durbey, S. D. 1970. Compound gamma, beta and F distributions, Metrika 16, 27 ?31.

[14] Ghitany, M. E., AL-Awadhi, F. A and Alkhalfan, L. A. 2007. Marshall-Olkin extended Lomax distribution and its applications to censored data, Communications in Statistics?Theory and Methods 36, 1855回1866.

[15] Glanzel, W. 2008. On some new bibliometric applications of statistics related to the h-index, Scientometrics 77, 187国196.

[16] Gupta, R. C., Ghitany, M. E. and Al-Mutairi, D. K. 2010. Estimation of reliability from Marshall-Olkin extended Lomax distributions, Journal of Statistical Computation and Simulation 80, 937回947.

[17] Harris, C. M. 1968. The Pareto distribution as a queue service descipline, Operations Research 16, 307?313.

[18] Hassan, A. S. and Al-Ghamdi, A. S. 2009. Optimum step stress accelerated life testing for Lomax distibution,

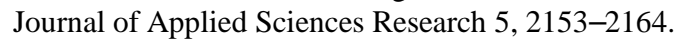

[19] Holland, O., Golaup, A. and Aghvami, A. H. Traffic characteristics of aggregated module downloads for mobile terminal recon?guration, IEE proceedings on Communications 135, 683 ? 690 . 
[20] Johnson, N. L., Kotz, S. and Balakrishnan, N. 1994 Contineous Univariate Distributions: Vol. 1, 2nd edition (Wiley, New York).

[21] Kareema, A. K. and Mohommad, A. B. 2013. Exponential Pareto Distribution, Mathematical Theory and Modeling, 2224-5804.

[22] Lee, M-Y. and Lim, E-K. 2009. Characterization of the Lomax, exponential and Pareto distributions by conditional expectations of recoed values, Journal of the Chungcheong Mathematical Society 22, 149?153.

[23] Lemonte, A. J. and Cordeiro, G. M. 2013. An extended Lomax distribution, Statistics 47, 800]816.
[24] Lomax, K. S. 1954. Business failures: Another example of the analysis of failure data, Journal of the American Statistical Association 49, 847]852.

[25] Murthy, D. N. P. Xie, M. and Jiang, R. 2004. Weibull Models (Wiley).

[26] Ramos, M. W. A., Marinho, P. R. D., da Silva R. V. and Cordeiro, G. M. 2013. The exponentiated Lomax Poisson distribution with an application to lifetime data, Advances and Applications in Statistics 34, 1079135.

[27] Tadikamalla, P. R. 1980. A look at the Burr and realted distributions, International Statistical Review 48, 337@344. 